\title{
Ultrasonographic Assessment of Renal Perfusion in Bitches With Mammary Carcinoma Treated With Long-Term Carprofen
}

Cristhian Vargas Estrada ( $\sim$ crisvaresmvz@gmail.com )

Sao Paulo State University

Bruna Fernanda Firmo

Sao Paulo State University

Daniele Belchior Vela

Sao Paulo State University

Marjury Cristina Maronezi

Sao Paulo State University

Ricardo Andrés Ramirez Uscategui

Universidade Federal dos Vales do Jequitinhonha e Mucuri

\section{Beatriz Gasser}

Sao Paulo State University

Marcus Antônio Rossi Feliciano

Universidade Federal de Santa Maria

Letícia Pavan

Sao Paulo State University

Luiz Paulo Nogueira Aires

Sao Paulo State University

Gabriela Piovan Lima

Sao Paulo State University

Andrigo Barboza De Nardi

Sao Paulo State University

\section{Research Article}

Keywords: mammary neoplasm, NSAIDs, renal perfusion, ultrasonography

Posted Date: February 18th, 2021

DOI: https://doi.org/10.21203/rs.3.rs-199333/v1 
License: (c) (i) This work is licensed under a Creative Commons Attribution 4.0 International License. Read Full License 


\section{Abstract}

Objective. The aim of this study was to evaluate renal hemodynamics, routine clinical and laboratory parameters used to estimate renal function, and clinical evolution during six months in bitches with mammary carcinomas that underwent mastectomy and were treated (TG) or not (CG) with carprofen for three months after surgery.

Methods. Twenty-six bitches with mammary neoplasia were equally distributed into TG that received carprofen $4.4 \mathrm{mg} / \mathrm{kg} /$ day for 90 days and CG that did not receive anti-inflammatory medication. Renal artery Doppler flowmetry, contrast-enhanced ultrasound (CEUS) of renal parenchyma, haematological, biochemical and clinical analyses were obtained once a month. These data were compared between groups and moments via analysis of variance (ANOVA) in a completely randomized design with repeated measures $(P<0.05)$.

Results. On B-mode ultrasound, the area of the renal artery was greater $(P=0.0003)$ in the TG. Regarding laboratory findings, haematocrit and haemoglobin were similar in both groups, showing a significant and gradual increase after three months of treatment; $M C V, M H C$, and $M C H C$ were increased $(P<0.05)$ and lymphocyte and band counts decreased $(P<0.05)$ in the TG. Regarding biochemical tests, ALT was the only parameter with a significant difference, being higher $(P=0.0272)$ in the treated group.

Conclusion. It can be concluded that the use of carprofen for 90 days causes minimal changes in renal perfusion, erythrocyte parameters and ALT activity, and reduces the proportion of blood inflammatory cells. Therefore, use of this medication can be carried out safely in patients who require auxiliary cancer treatment.

\section{Introduction}

The use of carprofen in vitro has been shown to suppress the proliferation and induce apoptosis of neoplastic cells [1], indicating that can be beneficial in the treatment of neoplasms with cyclooxygenase-2 (COX-2) expression. Doré et al. (2003) detected an overexpression of COX-2 in $56 \%$ of canine patients with mammary adenocarcinoma, suggesting that treatment with COX-2 inhibitors may be beneficial; and that COX-2 inhibitors reduce proliferation of neoplastic cells and may help to stall tumour growth in neoplasms [3].

However, prolonged use of nonsteroidal anti-inflammatory drugs (NSAIDs) may lead to serious renal side effects, mainly related to renal perfusion [4]. Due to the kidneys' importance for haemostasis, an adequate evaluation and strict control of renal changes in patients treated with carprofen for long periods of time are needed. Contrast-enhanced ultrasound (CEUS) and Doppler, which have shown promising results due to their accuracy and early identification of renal perfusion impairment $[5,6]$ and CEUS even allows amplification of Doppler signal, improving microcirculatory evaluation of the kidney [5]. 
Based on these precepts, the objective of this experimental trial was to evaluate renal hemodynamic using Doppler and CEUS techniques, routine clinical and laboratory parameters as markers of renal function, and clinical evolution during a six-month period in bitches with mammary carcinoma that underwent mastectomy and were treated (TG) or not treated (CG) with carprofen for three months after surgery.

\section{Results}

All patients evaluated by B-mode ultrasound showed kidney size and architecture within normal parameters, with homogeneous and hypoechoic aspect compared to splenic parenchyma, and preserved corticomedullary ratio, although a discrete loss of cortical thickness was detected in some patients. Only the renal artery area was increased $(P=0.0003)$ in CG during month 4 and in TG during months 1 to 6 . The others analysed parameters were similar between groups (Tables 1 to 3 ). 
Table 1

Mean \pm SD of B-mode ultrasound evaluation of the left kidney in bitches with mammary carcinoma treated (TG) or not treated (CG) with carprofen $4.4 \mathrm{mg} / \mathrm{kg} /$ day for 90 days and evaluated for 6 months.

\begin{tabular}{|c|c|c|c|c|c|c|}
\hline Variable & Moment & CG & TG & P-Group & P-Moment & P- Interaction \\
\hline \multirow[t]{7}{*}{ CMR } & MO & $0.91 \pm 0.16$ & $1.38 \pm 0.74$ & \multirow[t]{7}{*}{0.4593} & \multirow[t]{7}{*}{0.4038} & \multirow[t]{7}{*}{0.1095} \\
\hline & M1 & $0.95 \pm 0.17$ & $1.06 \pm 0.41$ & & & \\
\hline & M2 & $1.01 \pm 0.19$ & $0.92 \pm 0.26$ & & & \\
\hline & M3 & $1.01 \pm 0.22$ & $1.13 \pm 0.46$ & & & \\
\hline & M4 & $0.91 \pm 0.23$ & $1.02 \pm 0.38$ & & & \\
\hline & M5 & $0.77 \pm 0.16$ & $0.90 \pm 0.15$ & & & \\
\hline & M6 & $1.15 \pm 0.10$ & $0.97 \pm 0.09$ & & & \\
\hline \multirow[t]{7}{*}{$\mathrm{RA}\left(\mathrm{cm}^{2}\right)$} & Mo & $0.12 \pm 0.09$ & $0.09 \pm 0.01$ & \multirow[t]{7}{*}{0.6730} & \multirow[t]{7}{*}{$0.0002^{\star}$} & \multirow[t]{7}{*}{$0.0003^{\star}$} \\
\hline & M1 & $0.15 \pm 0.08$ & $0.25 \pm 0.15$ & & & \\
\hline & M2 & $0.18 \pm 0.12$ & $0.15 \pm 0.08$ & & & \\
\hline & M3 & $0.19 \pm 0.11$ & $0.16 \pm 0.10$ & & & \\
\hline & M4 & $0.21 \pm 0.03$ & $0.18 \pm 0.12$ & & & \\
\hline & M5 & $0.18 \pm 0.01$ & $0.15 \pm 0.10$ & & & \\
\hline & M6 & $0.20 \pm 0.07$ & $0.19 \pm 0.12$ & & & \\
\hline \multirow[t]{7}{*}{$\mathrm{RV}\left(\mathrm{cm}^{3}\right)$} & MO & $28.51 \pm 29.19$ & $24.93 \pm 19.03$ & \multirow[t]{7}{*}{0.5061} & \multirow[t]{7}{*}{0.8930} & \multirow[t]{7}{*}{0.8195} \\
\hline & M1 & $28.17 \pm 19.72$ & $35.76 \pm 22.64$ & & & \\
\hline & M2 & $29.32 \pm 18.32$ & $34.80 \pm 21.26$ & & & \\
\hline & M3 & $35.81 \pm 21.62$ & $31.38 \pm 20.88$ & & & \\
\hline & M4 & $37.91 \pm 17.62$ & $29.56 \pm 21.02$ & & & \\
\hline & M5 & $38.20 \pm 18.80$ & $26.55 \pm 17.55$ & & & \\
\hline & M6 & $39.10 \pm 27.90$ & $35.37 \pm 21.97$ & & & \\
\hline
\end{tabular}


Table 2

Mean \pm SD of Doppler evaluation of the left kidney in bitches with mammary carcinoma treated (TG) or not treated (CG) with carprofen $4.4 \mathrm{mg} / \mathrm{kg} / \mathrm{day}$ for 90 days and evaluated for 6 months.

\begin{tabular}{|c|c|c|c|c|c|c|}
\hline Variable & Moment & CG & TG & P-Group & P-Moment & P- Interaction \\
\hline \multirow[t]{7}{*}{ SV } & MO & $99.8 \pm 22.4$ & $114 \pm 6.69$ & \multirow[t]{7}{*}{0.3680} & \multirow[t]{7}{*}{0.8114} & \multirow[t]{7}{*}{0.9486} \\
\hline & M1 & $91.4 \pm 31.8$ & $109 \pm 31.0$ & & & \\
\hline & M2 & $87.4 \pm 32.8$ & $112 \pm 36.9$ & & & \\
\hline & M3 & $79.1 \pm 23.6$ & $104 \pm 31.3$ & & & \\
\hline & M4 & $89.1 \pm 39.9$ & $109 \pm 31.8$ & & & \\
\hline & M5 & $92.3 \pm 24.4$ & $98.6 \pm 17.1$ & & & \\
\hline & M6 & $94.7 \pm 44.7$ & $96.6 \pm 9.15$ & & & \\
\hline \multirow[t]{7}{*}{ DV } & MO & $27.9 \pm 6.57$ & $32.0 \pm 1.77$ & \multirow[t]{7}{*}{0.2049} & \multirow[t]{7}{*}{0.8626} & \multirow[t]{7}{*}{0.7478} \\
\hline & M1 & $29.6 \pm 13.4$ & $37.0 \pm 12.7$ & & & \\
\hline & M2 & $28.5 \pm 16.4$ & $35.1 \pm 16.0$ & & & \\
\hline & M3 & $25.9 \pm 9.42$ & $34.1 \pm 15.6$ & & & \\
\hline & M4 & $37.5 \pm 26.5$ & $31.8 \pm 14.2$ & & & \\
\hline & M5 & $27.3 \pm 14.1$ & $28.9 \pm 6.23$ & & & \\
\hline & M6 & $29.1 \pm 5.49$ & $32.4 \pm 4.36$ & & & \\
\hline \multirow[t]{7}{*}{ RI } & MO & $0.71 \pm 0.04$ & $0.72 \pm 0.02$ & \multirow[t]{7}{*}{0.9990} & \multirow[t]{7}{*}{0.2733} & \multirow[t]{7}{*}{0.3679} \\
\hline & M1 & $0.67 \pm 0.08$ & $0.65 \pm 0.08$ & & & \\
\hline & M2 & $0.68 \pm 0.08$ & $0.68 \pm 0.08$ & & & \\
\hline & M3 & $0.67 \pm 0.06$ & $0.67 \pm 0.06$ & & & \\
\hline & M4 & $0.60 \pm 0.12$ & $0.70 \pm 0.09$ & & & \\
\hline & M5 & $0.71 \pm 0.09$ & $0.70 \pm 0.03$ & & & \\
\hline & M6 & $0.65 \pm 0.11$ & $0.66 \pm 0.05$ & & & \\
\hline \multirow[t]{4}{*}{ IP } & MO & $1.78 \pm 0.31$ & $1.60 \pm 0.16$ & \multirow[t]{4}{*}{0.8629} & \multirow[t]{4}{*}{0.1354} & \multirow[t]{4}{*}{0.4421} \\
\hline & M1 & $1.62 \pm 0.43$ & $1.56 \pm 0.57$ & & & \\
\hline & M2 & $1.55 \pm 0.57$ & $1.67 \pm 0.64$ & & & \\
\hline & M3 & $1.88 \pm 0.70$ & $1.57 \pm 0.38$ & & & \\
\hline
\end{tabular}




\begin{tabular}{|c|c|c|c|c|c|c|}
\hline Variable & Moment & CG & TG & P-Group & P-Moment & P- Interaction \\
\hline & M4 & $1.47 \pm 1.15$ & $1.83 \pm 0.67$ & & & \\
\hline & M5 & $1.99 \pm 1.07$ & $1.88 \pm 0.58$ & & & \\
\hline & M6 & $1.31 \pm 0.45$ & $1.43 \pm 0.27$ & & & \\
\hline \multirow[t]{7}{*}{$\mathrm{RBF}(\mathrm{ml} / \mathrm{min})$} & Mo & $1.55 \pm 0.73$ & $1.40 \pm 0.17$ & 0.2572 & 0.0526 & 0.1245 \\
\hline & M1 & $1.35 \pm 0.33$ & $2.26 \pm 0.88$ & & & \\
\hline & M2 & $1.61 \pm 1.25$ & $1.56 \pm 0.62$ & & & \\
\hline & M3 & $1.09 \pm 0.53$ & $1.39 \pm 0.67$ & & & \\
\hline & M4 & $1.66 \pm 1.09$ & $1.62 \pm 0.61$ & & & \\
\hline & M5 & $0.99 \pm 0.37$ & $1.30 \pm 0.60$ & & & \\
\hline & M6 & $1.61 \pm 0.63$ & $1.37 \pm 0.19$ & & & \\
\hline
\end{tabular}


Table 3

Mean \pm SD of CEUS evaluation of the left kidney in bitches with mammary carcinoma treated (TG) or not treated (CG) with carprofen $4.4 \mathrm{mg} / \mathrm{kg} /$ day for 90 days and evaluated for 6 months.

\begin{tabular}{|c|c|c|c|c|c|c|}
\hline Variable & Moment & CG & TG & $\begin{array}{l}\text { P- } \\
\text { Group }\end{array}$ & $\begin{array}{l}\text { P- } \\
\text { Moment }\end{array}$ & $\begin{array}{l}\text { P- } \\
\text { Interaction }\end{array}$ \\
\hline \multirow[t]{7}{*}{ CortPI (\%) } & MO & $22.8 \pm 7.99$ & $21.3 \pm 1.88$ & \multirow[t]{7}{*}{0.2619} & \multirow[t]{7}{*}{0.4152} & \multirow[t]{7}{*}{0.7322} \\
\hline & M1 & $23.1 \pm 5.25$ & $22.3 \pm 9.14$ & & & \\
\hline & M2 & $25.4 \pm 8.42$ & $26.1 \pm 6.11$ & & & \\
\hline & M3 & $21.1 \pm 3.6$ & $25.2 \pm 4.36$ & & & \\
\hline & M4 & $23.7 \pm 2.73$ & $27.4 \pm 19.1$ & & & \\
\hline & M5 & $27.4 \pm 11.8$ & $23.3 \pm 6.6$ & & & \\
\hline & M6 & $23.7 \pm 12.6$ & $40.3 \pm 36.9$ & & & \\
\hline \multirow{7}{*}{$\begin{array}{l}\text { CortTmT } \\
\text { (s) }\end{array}$} & MO & $29.3 \pm 16.2$ & $19.7 \pm 1.77$ & \multirow[t]{7}{*}{0.3399} & \multirow[t]{7}{*}{0.5822} & \multirow[t]{7}{*}{0.533} \\
\hline & M1 & $24.9 \pm 4.42$ & $25.6 \pm 14.5$ & & & \\
\hline & M2 & $25.3 \pm 9.76$ & $30.7 \pm 13.3$ & & & \\
\hline & M3 & $24.1 \pm 3.95$ & $25.5 \pm 10.6$ & & & \\
\hline & M4 & $20.5 \pm 7.56$ & $27.1 \pm 7.24$ & & & \\
\hline & M5 & $22.1 \pm 13.9$ & $35.0 \pm 19.3$ & & & \\
\hline & M6 & $29.2 \pm 11.2$ & $33.0 \pm 9.93$ & & & \\
\hline \multirow[t]{7}{*}{ CortAUC } & MO & $963 \pm 1038$ & $548 \pm 80.1$ & \multirow[t]{7}{*}{0.2695} & \multirow[t]{7}{*}{0.6137} & \multirow[t]{7}{*}{0.7469} \\
\hline & M1 & $734 \pm 219$ & $815 \pm 752$ & & & \\
\hline & M2 & $786 \pm 381$ & $1004 \pm 555$ & & & \\
\hline & M3 & $655 \pm 152$ & $892 \pm 476$ & & & \\
\hline & M4 & $545 \pm 194$ & $863 \pm 481$ & & & \\
\hline & M5 & $734 \pm 659$ & $1024 \pm 510$ & & & \\
\hline & M6 & $815 \pm 472$ & $1319 \pm 862$ & & & \\
\hline \multirow{2}{*}{$\begin{array}{l}\text { (a) Cort } \\
(\% / s)\end{array}$} & MO & $1.80 \pm 0.83$ & $1.86 \pm 0.94$ & \multirow[t]{2}{*}{0.5991} & \multirow[t]{2}{*}{0.7909} & \multirow[t]{2}{*}{0.5253} \\
\hline & M1 & $1.68 \pm 0.39$ & $1.7 \pm 0.79$ & & & \\
\hline \multicolumn{7}{|c|}{$\begin{array}{l}\text { CortPI: cortical peak intensity; CortTmT: cortical mean time of transmission; CortAUC: cortical mean } \\
\text { area under the curve; (a) Cort: cortical wash-in slope; (b) Cort; cortical wash-out slope; MedPI: } \\
\text { medullary peak intensity; MedTp: medullary time until peak intensity; MedTmT: medullary mean time } \\
\text { of transmission; MedAUC: medullary area under the curve; (a) Med: medullary wash-in slope; (b) Med: } \\
\text { medullary wash-out slope. }\end{array}$} \\
\hline
\end{tabular}




\begin{tabular}{|c|c|c|c|c|c|c|}
\hline Variable & Moment & CG & TG & $\begin{array}{l}\text { P- } \\
\text { Group }\end{array}$ & $\begin{array}{l}\text { P- } \\
\text { Moment }\end{array}$ & $\begin{array}{l}\text { P- } \\
\text { Interaction }\end{array}$ \\
\hline & M2 & $2.09 \pm 1.13$ & $1.89 \pm 0.95$ & & & \\
\hline & M3 & $1.75 \pm 0.63$ & $2.31 \pm 0.76$ & & & \\
\hline & M4 & $1.87 \pm 0.71$ & $1.91 \pm 0.93$ & & & \\
\hline & M5 & $1.93 \pm 0.55$ & $1.73 \pm 0.78$ & & & \\
\hline & M6 & $1.37 \pm 1.12$ & $2.30 \pm 1.80$ & & & \\
\hline \multirow{7}{*}{$\begin{array}{l}\text { (b) Cort } \\
(\% / s)\end{array}$} & MO & $2.22 \pm 1.36$ & $3.57 \pm 1.69$ & \multirow[t]{7}{*}{0.911} & \multirow[t]{7}{*}{0.3934} & \multirow[t]{7}{*}{0.4017} \\
\hline & M1 & $2.58 \pm 1.25$ & $3.25 \pm 2.28$ & & & \\
\hline & M2 & $3.32 \pm 2.38$ & $3.14 \pm 2.15$ & & & \\
\hline & M3 & $2.22 \pm 1.04$ & $2.76 \pm 2.06$ & & & \\
\hline & M4 & $4.9 \pm 3.36$ & $3.98 \pm 4.99$ & & & \\
\hline & M5 & $5.69 \pm 2.59$ & $2.38 \pm 2.7$ & & & \\
\hline & M6 & $2.95 \pm 1.45$ & $2.66 \pm 1.99$ & & & \\
\hline \multirow[t]{7}{*}{ MedPI (\%) } & Mo & $22.1 \pm 3.71$ & $25.0 \pm 10.3$ & \multirow[t]{7}{*}{0.0544} & \multirow[t]{7}{*}{0.5365} & \multirow[t]{7}{*}{0.1343} \\
\hline & M1 & $24.8 \pm 8.75$ & $25.4 \pm 8.41$ & & & \\
\hline & M2 & $24.9 \pm 6.1$ & $30.8 \pm 7.01$ & & & \\
\hline & M3 & $18.2 \pm 5.18$ & $30.3 \pm 7.64$ & & & \\
\hline & M4 & $24.2 \pm 8.31$ & $23.0 \pm 11.6$ & & & \\
\hline & M5 & $32.8 \pm 5.41$ & $26.2 \pm 9.45$ & & & \\
\hline & M6 & $31.6 \pm 9.29$ & $27.2 \pm 8.54$ & & & \\
\hline \multirow[t]{5}{*}{ MedTp (s) } & MO & $46.3 \pm 14.2$ & $52.8 \pm 4.29$ & \multirow[t]{5}{*}{0.8617} & \multirow[t]{5}{*}{0.6705} & \multirow[t]{5}{*}{0.9907} \\
\hline & M1 & $51.9 \pm 8.82$ & $51.8 \pm 21.7$ & & & \\
\hline & M2 & $46.1 \pm 22.5$ & $47.3 \pm 12.6$ & & & \\
\hline & M3 & $48.4 \pm 16.9$ & $48.7 \pm 14.9$ & & & \\
\hline & M4 & $52.7 \pm 7.07$ & $48.8 \pm 9.69$ & & & \\
\hline $\begin{array}{l}\text { CortPI: corti } \\
\text { area under } \\
\text { medullary p } \\
\text { of transmis } \\
\text { medullary }\end{array}$ & $\begin{array}{l}\text { peak inte } \\
\text { curve; (a) } \\
\text { k intensity } \\
\text { n; MedAU } \\
\text { h-out slop }\end{array}$ & $\begin{array}{l}\text { CortTmT: ce } \\
\text { cortical was } \\
\text { dTp: medulla } \\
\text { edullary area }\end{array}$ & $\begin{array}{l}\text { al mean time } \\
\text { slope; (b) Co } \\
\text { me until peak } \\
\text { ler the curve; }\end{array}$ & $\begin{array}{l}\text { nsmissio } \\
\text { ical wasł } \\
\text { ity; Med7 } \\
\text { d: medull }\end{array}$ & $\begin{array}{l}\text { CortAUC: c } \\
\text { ut slope; } \\
\text { T: medulla } \\
\text { y wash-in }\end{array}$ & $\begin{array}{l}\text { tical mean } \\
\text { dPI: } \\
\text { mean time } \\
\text { pe; (b) Med: }\end{array}$ \\
\hline
\end{tabular}




\begin{tabular}{|c|c|c|c|c|c|c|}
\hline Variable & Moment & CG & TG & $\begin{array}{l}\text { P- } \\
\text { Group }\end{array}$ & $\begin{array}{l}\text { P- } \\
\text { Moment }\end{array}$ & $\begin{array}{l}\text { P- } \\
\text { Interaction }\end{array}$ \\
\hline & M5 & $59.8 \pm 4.05$ & $54.8 \pm 9.7$ & & & \\
\hline & M6 & $57.7 \pm 29.7$ & $53.6 \pm 11.1$ & & & \\
\hline \multirow{7}{*}{$\begin{array}{l}\text { MedTmT } \\
\text { (s) }\end{array}$} & Mo & $58.8 \pm 15.4$ & $70.2 \pm 0.19$ & 0.9923 & 0.7301 & 0.9775 \\
\hline & M1 & $65.7 \pm 10.1$ & $63.2 \pm 21.9$ & & & \\
\hline & M2 & $60.9 \pm 27.2$ & $57.0 \pm 14.1$ & & & \\
\hline & M3 & $62.4 \pm 15.0$ & $59.9 \pm 17.3$ & & & \\
\hline & M4 & $63.5 \pm 7.08$ & $61.6 \pm 10.7$ & & & \\
\hline & M5 & $70.0 \pm 6.42$ & $72.5 \pm 20.5$ & & & \\
\hline & M6 & $64.1 \pm 26.1$ & $64.2 \pm 11.0$ & & & \\
\hline \multirow[t]{7}{*}{ MedAUC } & Mo & $1267 \pm 413$ & $1573 \pm 365$ & 0.5217 & 0.6217 & 0.6358 \\
\hline & M1 & $1602 \pm 471$ & $1568 \pm 780$ & & & \\
\hline & M2 & $1618 \pm 1116$ & $1579 \pm 478$ & & & \\
\hline & M3 & $1171 \pm 458$ & $1780 \pm 848$ & & & \\
\hline & M4 & $1386 \pm 474$ & $1471 \pm 802$ & & & \\
\hline & M5 & $1964 \pm 44.3$ & $1781 \pm 511$ & & & \\
\hline & M6 & $2232 \pm 1700$ & $1694 \pm 912$ & & & \\
\hline \multirow{7}{*}{$\begin{array}{l}\text { (a) Med } \\
(\% / s)\end{array}$} & MO & $0.54 \pm 0.25$ & $0.48 \pm 0.23$ & 0.7263 & 0.1773 & 0.6626 \\
\hline & M1 & $0.49 \pm 0.18$ & $0.52 \pm 0.13$ & & & \\
\hline & M2 & $0.89 \pm 0.85$ & $0.67 \pm 0.17$ & & & \\
\hline & M3 & $0.41 \pm 0.16$ & $0.65 \pm 0.19$ & & & \\
\hline & M4 & $0.45 \pm 0.13$ & $0.47 \pm 0.22$ & & & \\
\hline & M5 & $0.55 \pm 0.13$ & $0.50 \pm 0.24$ & & & \\
\hline & M6 & $0.67 \pm 0.45$ & $0.52 \pm 0.17$ & & & \\
\hline $\begin{array}{l}\text { (b) Med } \\
(\% / s)\end{array}$ & MO & $2.07 \pm 0.85$ & $1.41 \pm 0.26$ & 0.9171 & 0.7117 & 0.6999 \\
\hline \multicolumn{7}{|c|}{$\begin{array}{l}\text { CortPI: cortical peak intensity; CortTmT: cortical mean time of transmission; CortAUC: cortical mean } \\
\text { area under the curve; (a) Cort: cortical wash-in slope; (b) Cort; cortical wash-out slope; MedPI: } \\
\text { medullary peak intensity; MedTp: medullary time until peak intensity; MedTmT: medullary mean time } \\
\text { of transmission; MedAUC: medullary area under the curve; (a) Med: medullary wash-in slope; (b) Med: } \\
\text { medullary wash-out slope. }\end{array}$} \\
\hline
\end{tabular}




\begin{tabular}{|c|c|c|c|c|c|c|}
\hline Variable & Moment & CG & TG & $\begin{array}{l}\text { P- } \\
\text { Group }\end{array}$ & $\begin{array}{l}\text { P- } \\
\text { Moment }\end{array}$ & $\begin{array}{l}\text { P- } \\
\text { Interaction }\end{array}$ \\
\hline & M1 & $2.17 \pm 1.29$ & $2.43 \pm 1.17$ & & & \\
\hline & M2 & $2.56 \pm 2.45$ & $3.63 \pm 1.56$ & & & \\
\hline & M3 & $1.41 \pm 0.60$ & $3.22 \pm 1.89$ & & & \\
\hline & M4 & $2.36 \pm 1.08$ & $2.20 \pm 1.67$ & & & \\
\hline & M5 & $3.44 \pm 1.48$ & $2.20 \pm 1.90$ & & & \\
\hline & M6 & $2.77 \pm 0.84$ & $2.66 \pm 0.57$ & & & \\
\hline \multicolumn{7}{|c|}{$\begin{array}{l}\text { CortPI: cortical peak intensity; CortTmT: cortical mean time of transmission; CortAUC: cortical mean } \\
\text { area under the curve; (a) Cort: cortical wash-in slope; (b) Cort; cortical wash-out slope; MedPI: } \\
\text { medullary peak intensity; MedTp: medullary time until peak intensity; MedTmT: medullary mean time } \\
\text { of transmission; MedAUC: medullary area under the curve; (a) Med: medullary wash-in slope; (b) Med } \\
\text { medullary wash-out slope. }\end{array}$} \\
\hline
\end{tabular}

Regarding laboratory findings (Tables 4 and 5), there was no difference between groups for haematocrit and haemoglobin, but both parameters were significantly and gradually increased after the third month $(P<0.05)$. On the other hand, $M C V, M C H$, and $M C H C$ were higher in treated animals $(P<0.05)$ and similar between moments $(P>0.05)$. The bands and lymphocytes count were higher in $C G$ compared to $T G(P<$ $0.05)$ and similar between moments $(P>0.05)$. The neutrophils/lymphocytes ratio was smaller in TG during the second and third months, while in month 6 it was smaller in CG $(P<0.05)$. Also, ALT was higher in TG $(P<0.05)$ and similar between moments $(P>0.05)$. The remaining evaluated parameters showed no significant differences related to treatments or moments. 
Table 4

Mean \pm SD of hematologic findings in bitches with mammary carcinoma treated (TG) or not treated (CG) with carprofen $4.4 \mathrm{mg} / \mathrm{kg} / \mathrm{day}$ for 90 days and evaluated for 6 months.

\begin{tabular}{|c|c|c|c|c|c|c|}
\hline Variable & Moment & CG & TG & $\begin{array}{l}\text { P- } \\
\text { Group }\end{array}$ & $\begin{array}{l}\text { P- } \\
\text { Moment }\end{array}$ & $\begin{array}{l}\text { P- } \\
\text { Interaction }\end{array}$ \\
\hline \multirow[t]{7}{*}{$\mathrm{HB}(\mathrm{g} / \mathrm{dL})$} & MO & $12.7 \pm 4.04^{a}$ & $14.4 \pm 1.84^{a}$ & \multirow[t]{7}{*}{0.146} & \multirow[t]{7}{*}{$0.0338^{*}$} & \multirow[t]{7}{*}{0.8182} \\
\hline & M1 & $15.5 \pm 2.71^{a}$ & $15.4 \pm 3.23^{a}$ & & & \\
\hline & M2 & $16.0 \pm 2.79^{a}$ & $15.7 \pm 3.41^{a}$ & & & \\
\hline & M3 & $16.5 \pm 3.12^{b}$ & $16.8 \pm 3.38^{b}$ & & & \\
\hline & M4 & $16.74 \pm 3^{b}$ & $17.4 \pm 2.62^{b}$ & & & \\
\hline & M5 & $16.0 \pm 1.77^{\mathrm{b}}$ & $17.1 \pm 2.47^{b}$ & & & \\
\hline & M6 & $15.8 \pm 2.58^{b}$ & $18.8 \pm 1.83^{b}$ & & & \\
\hline \multirow[t]{7}{*}{ HT (\%) } & MO & $36.5 \pm 11.2^{\mathrm{a}}$ & $42.2 \pm 5.16^{\mathrm{a}}$ & \multirow[t]{7}{*}{0.3048} & \multirow[t]{7}{*}{$0.0287^{*}$} & \multirow[t]{7}{*}{0.8649} \\
\hline & M1 & $45 \pm 7.31^{a}$ & $44.6 \pm 8.39^{a}$ & & & \\
\hline & M2 & $46.5 \pm 6.97^{a}$ & $44.4 \pm 7.84^{a}$ & & & \\
\hline & M3 & $47.4 \pm 8.43^{b}$ & $48.4 \pm 10.2^{b}$ & & & \\
\hline & M4 & $48.2 \pm 7.28^{b}$ & $48.8 \pm 7.33^{b}$ & & & \\
\hline & M5 & $47 \pm 5.37^{b}$ & $48.2 \pm 6.8^{b}$ & & & \\
\hline & M6 & $46.4 \pm 7.57^{b}$ & $52.4 \pm 4.98^{b}$ & & & \\
\hline \multirow[t]{7}{*}{$\mathrm{MCV}(\mathrm{fL})$} & MO & $65.9 \pm 3.08^{a}$ & $69.3 \pm 1.75^{b}$ & \multirow[t]{7}{*}{$0.0082^{\star}$} & \multirow[t]{7}{*}{0.5247} & \multirow[t]{7}{*}{0.9549} \\
\hline & M1 & $68.4 \pm 4.09^{a}$ & $70.0 \pm 3.47^{b}$ & & & \\
\hline & M2 & $68.2 \pm 4.56^{a}$ & $68.5 \pm 3.22^{b}$ & & & \\
\hline & M3 & $68.4 \pm 4.76^{a}$ & $70.4 \pm 3.22^{b}$ & & & \\
\hline & M4 & $68.0 \pm 4.82^{a}$ & $71.2 \pm 2.81^{b}$ & & & \\
\hline & M5 & $69.4 \pm 4.49^{a}$ & $70.8 \pm 2.05^{b}$ & & & \\
\hline & M6 & $69.6 \pm 4.13^{\mathrm{a}}$ & $71.2 \pm 2.92^{b}$ & & & \\
\hline
\end{tabular}

$\mathrm{HB}$ : hemoglobin; $\mathrm{HT}$ : hematocrit; $\mathrm{MCV}$ : mean corpuscular volume; $\mathrm{MCH}$ : mean corpuscular hemoglobin; MCHC: mean corpuscular hemoglobin concentration; BTS: bands; LFT: lymphocytes; RNL: neutrophil/lymphocyte ratio. 


\begin{tabular}{|c|c|c|c|c|c|c|}
\hline Variable & Moment & CG & TG & $\begin{array}{l}\text { P- } \\
\text { Group }\end{array}$ & $\begin{array}{l}\text { P- } \\
\text { Moment }\end{array}$ & $\begin{array}{l}\text { P- } \\
\text { Interaction }\end{array}$ \\
\hline \multirow[t]{7}{*}{$\mathrm{MCH}(\mathrm{pg})$} & MO & $22.8 \pm 1.28^{a}$ & $23.6 \pm 0.46^{b}$ & \multirow[t]{7}{*}{$0.0002 *$} & \multirow[t]{7}{*}{0.3688} & \multirow[t]{7}{*}{0.7475} \\
\hline & M1 & $23.6 \pm 1.51^{a}$ & $24.1 \pm 1.96^{b}$ & & & \\
\hline & M2 & $23.4 \pm 1.43^{a}$ & $24.1 \pm 2.25^{b}$ & & & \\
\hline & M3 & $23.7 \pm 1.69^{a}$ & $24.5 \pm 1.24^{b}$ & & & \\
\hline & M4 & $23.4 \pm 1.81^{\mathrm{a}}$ & $25.4 \pm 0.96^{b}$ & & & \\
\hline & M5 & $23.7 \pm 1.18^{a}$ & $25.2 \pm 0.72^{b}$ & & & \\
\hline & M6 & $23.7 \pm 1.07^{a}$ & $25.6 \pm 1.19^{b}$ & & & \\
\hline \multirow[t]{7}{*}{$\mathrm{MCHC}(\mathrm{g} / \mathrm{dL})$} & MO & $34.6 \pm 1.11^{\mathrm{a}}$ & $34.0 \pm 0.18^{b}$ & \multirow[t]{7}{*}{$0.0285^{\star}$} & \multirow[t]{7}{*}{0.8232} & \multirow[t]{7}{*}{0.3192} \\
\hline & M1 & $34.5 \pm 1.02^{\mathrm{a}}$ & $34.4 \pm 1.68^{b}$ & & & \\
\hline & M2 & $34.3 \pm 1.49^{a}$ & $35.2 \pm 2.13^{b}$ & & & \\
\hline & M3 & $34.7 \pm 1.64^{a}$ & $34.8 \pm 1.02^{b}$ & & & \\
\hline & M4 & $34.5 \pm 1.98^{a}$ & $35.7 \pm 0.68^{b}$ & & & \\
\hline & M5 & $34.1 \pm 0.71^{a}$ & $35.5 \pm 0.61^{b}$ & & & \\
\hline & M6 & $34.1 \pm 1.26^{a}$ & $35.9 \pm 0.5^{\mathrm{b}}$ & & & \\
\hline \multirow[t]{7}{*}{ BTS $\left(x 10^{3} / u L\right)$} & MO & $0.42 \pm 0.78^{a}$ & $0 \pm 0^{b}$ & \multirow[t]{7}{*}{$0.0459 *$} & \multirow[t]{7}{*}{0.6567} & \multirow[t]{7}{*}{0.5487} \\
\hline & M1 & $0.63 \pm 1.28^{a}$ & $0.07 \pm 0.27^{b}$ & & & \\
\hline & M2 & $0.09 \pm 0.30^{a}$ & $0 \pm 0^{b}$ & & & \\
\hline & M3 & $0.63 \pm 1.02^{a}$ & $0.12 \pm 0.35^{\mathrm{b}}$ & & & \\
\hline & M4 & $0.5 \pm 0.75^{a}$ & $0.28 \pm 0.48^{b}$ & & & \\
\hline & M5 & $0 \pm 0^{a}$ & $0.4 \pm 0.54^{b}$ & & & \\
\hline & M6 & $0.2 \pm 0.44^{a}$ & $0.16 \pm 0.40^{\mathrm{b}}$ & & & \\
\hline LFT (\%) & MO & $17.8 \pm 7.4^{\mathrm{a}}$ & $15 \pm 1.41^{b}$ & $0.0201 *$ & 0.4954 & 0.2892 \\
\hline $\begin{array}{l}\text { HB: hemoglobi } \\
\text { hemoglobin; } \\
\text { RNL: neutroph }\end{array}$ & $\begin{array}{l}\text { T: hema } \\
\text { : mean } \\
\text { mphocy }\end{array}$ & $\begin{array}{l}\text { t; MCV: mea } \\
\text { uscular hem } \\
\text { io. }\end{array}$ & $\begin{array}{l}\text { scular volu } \\
\text { concentra }\end{array}$ & $\begin{array}{l}\text { CH: me } \\
\text { TS: bar }\end{array}$ & $\begin{array}{l}\text { orpuscl } \\
\text { FT. Ivm }\end{array}$ & te \\
\hline
\end{tabular}




\begin{tabular}{|c|c|c|c|c|c|c|}
\hline Variable & Moment & CG & TG & $\begin{array}{l}\text { P- } \\
\text { Group }\end{array}$ & $\begin{array}{l}\text { P- } \\
\text { Moment }\end{array}$ & $\begin{array}{l}\text { P- } \\
\text { Interaction }\end{array}$ \\
\hline & M1 & $23.3 \pm 7.02^{\mathrm{a}}$ & $17.6 \pm 5.6^{b}$ & & & \\
\hline & M2 & $19.0 \pm 4.23^{a}$ & $15.6 \pm 6.99^{b}$ & & & \\
\hline & M3 & $21.3 \pm 7.89^{a}$ & $13.5 \pm 8.19^{b}$ & & & \\
\hline & M4 & $18 \pm 7.95^{a}$ & $20.2 \pm 7.99^{b}$ & & & \\
\hline & M5 & $19.2 \pm 4.32^{\mathrm{a}}$ & $15 \pm 5.66^{b}$ & & & \\
\hline & M6 & $14.4 \pm 8.17^{a}$ & $16.8 \pm 4.36^{\mathrm{b}}$ & & & \\
\hline \multirow[t]{7}{*}{ RNL } & MO & $4.80 \pm 2.67^{a}$ & $5.085 \pm 1.092^{a}$ & \multirow[t]{7}{*}{$0.0125^{\star}$} & \multirow[t]{7}{*}{$0.0356 *$} & \multirow[t]{7}{*}{$0.00719 *$} \\
\hline & M1 & $3.193 \pm 1.169^{a}$ & $4.798 \pm 2.163^{a}$ & & & \\
\hline & M2 & $4.018 \pm 1.331 a$ & $5.98 \pm 3.06^{b}$ & & & \\
\hline & M3 & $3.766 \pm 1.683^{a}$ & $6.50 \pm 3.22^{b}$ & & & \\
\hline & M4 & $6.31 \pm 6.50^{\mathrm{a}}$ & $4.197 \pm 2.379^{a}$ & & & \\
\hline & M5 & $4.023 \pm 0.946^{a}$ & $5.89 \pm 2.47^{a}$ & & & \\
\hline & M6 & $8.43 \pm 6.28^{b}$ & $4.667 \pm 1.451^{\mathrm{a}}$ & & & \\
\hline
\end{tabular}


Table 5

Mean \pm SD of serum and urinary biochemistry findings in bitches with mammary carcinoma treated (TG) or not treated (CG) with $4.4 \mathrm{mg} / \mathrm{kg} /$ day of carprofen for 90 days and evaluated for 6 months.

\begin{tabular}{|c|c|c|c|c|c|c|}
\hline Variable & Moment & CG & TG & P-Group & P-Moment & P-Interaction \\
\hline \multirow[t]{7}{*}{ CREA (mg/dL) } & MO & $0.95 \pm 0.50$ & $0.95 \pm 0.21$ & \multirow[t]{7}{*}{0.9367} & \multirow[t]{7}{*}{0.7068} & \multirow[t]{7}{*}{0.9897} \\
\hline & M1 & $0.95 \pm 0.28$ & $1.00 \pm 0.24$ & & & \\
\hline & M2 & $0.91 \pm 0.24$ & $0.93 \pm 0.25$ & & & \\
\hline & M3 & $0.89 \pm 0.33$ & $0.93 \pm 0.17$ & & & \\
\hline & M4 & $0.97 \pm 0.33$ & $0.89 \pm 0.20$ & & & \\
\hline & M5 & $0.97 \pm 0.30$ & $0.86 \pm 0.25$ & & & \\
\hline & M6 & $0.77 \pm 0.23$ & $0.78 \pm 0.29$ & & & \\
\hline \multirow[t]{7}{*}{ UREA (mg/dL) } & M0 & $31.2 \pm 16.9$ & $26.5 \pm 4.95$ & \multirow[t]{7}{*}{0.1629} & \multirow[t]{7}{*}{0.2078} & \multirow[t]{7}{*}{0.6425} \\
\hline & M1 & $31.2 \pm 9.62$ & $34.9 \pm 10.6$ & & & \\
\hline & M2 & $33.5 \pm 11.5$ & $43.13 \pm 3$ & & & \\
\hline & M3 & $38.6 \pm 14.2$ & $35.7 \pm 10.6$ & & & \\
\hline & M4 & $32.8 \pm 8.13$ & $36.4 \pm 7.16$ & & & \\
\hline & M5 & $28 \pm 5.74$ & $32.6 \pm 10.6$ & & & \\
\hline & M6 & $28.2 \pm 9.26$ & $31.6 \pm 6.92$ & & & \\
\hline \multirow[t]{7}{*}{$\mathrm{ALT}(\mathrm{U} / \mathrm{L})$} & Mo & $42.3 \pm 30.5^{a}$ & $61.5 \pm 53^{b}$ & \multirow[t]{7}{*}{$0.0272^{\star}$} & \multirow[t]{7}{*}{0.7279} & \multirow[t]{7}{*}{0.9789} \\
\hline & M1 & $39.6 \pm 18.5^{a}$ & $50.5 \pm 36.5^{b}$ & & & \\
\hline & M2 & $39.2 \pm 16.0^{\mathrm{a}}$ & $52.6 \pm 33.1^{\mathrm{b}}$ & & & \\
\hline & M3 & $43.9 \pm 18.9^{a}$ & $61 \pm 30.6^{b}$ & & & \\
\hline & M4 & $33.2 \pm 6.94^{\mathrm{a}}$ & $48.1 \pm 21.7^{b}$ & & & \\
\hline & M5 & $38.2 \pm 23.4^{a}$ & $45.2 \pm 26.3^{b}$ & & & \\
\hline & M6 & $37.6 \pm 19.5^{a}$ & $37.3 \pm 13.2^{b}$ & & & \\
\hline \multirow[t]{3}{*}{ DEND } & M0 & $1034 \pm 19$ & $1012 \pm 3.54$ & \multirow[t]{3}{*}{0.9319} & \multirow[t]{3}{*}{0.9768} & \multirow[t]{3}{*}{0.1321} \\
\hline & M1 & $1028 \pm 7.78$ & $1027 \pm 11.7$ & & & \\
\hline & M2 & $1023 \pm 9.75$ & $1033 \pm 15.1$ & & & \\
\hline
\end{tabular}




\begin{tabular}{|c|c|c|c|c|c|c|}
\hline Variable & Moment & CG & TG & P-Group & P-Moment & P-Interaction \\
\hline & M3 & $1031 \pm 11.9$ & $1027 \pm 11$ & & & \\
\hline & M4 & $1030 \pm 11.6$ & $1032 \pm 13.1$ & & & \\
\hline & M5 & $1030 \pm 11.2$ & $1025 \pm 13.2$ & & & \\
\hline & M6 & $1026 \pm 11.9$ & $1032 \pm 12.9$ & & & \\
\hline \multirow[t]{7}{*}{$\mathrm{Ph}$} & MO & $6.16 \pm 0.87$ & $5.75 \pm 1.06$ & 0.3356 & 0.0921 & 0.754 \\
\hline & M1 & $6.68 \pm 1.48$ & $5.96 \pm 0.80$ & & & \\
\hline & M2 & $7.04 \pm 1.44$ & $6.5 \pm 1.28$ & & & \\
\hline & M3 & $6.40 \pm 1.2$ & $6.5 \pm 1.16$ & & & \\
\hline & M4 & $6.75 \pm 1.25$ & $6.78 \pm 1.15$ & & & \\
\hline & M5 & $5.3 \pm 0.44$ & $5.9 \pm 0.89$ & & & \\
\hline & M6 & $5.8 \pm 0.83$ & $5.91 \pm 1.42$ & & & \\
\hline \multirow[t]{7}{*}{ UPC } & MO & $0.55 \pm 0.73$ & $0.08 \pm 0.05$ & 0.2112 & 0.9103 & 0.9212 \\
\hline & M1 & $0.37 \pm 0.56$ & $0.28 \pm 0.33$ & & & \\
\hline & M2 & $0.36 \pm 0.54$ & $0.49 \pm 0.90$ & & & \\
\hline & M3 & $0.50 \pm 0.69$ & $0.24 \pm 0.14$ & & & \\
\hline & M4 & $0.66 \pm 1.01$ & $0.35 \pm 0.57$ & & & \\
\hline & M5 & $0.24 \pm 0.30$ & $0.13 \pm 0.10$ & & & \\
\hline & M6 & $0.39 \pm 0.47$ & $0.32 \pm 0.39$ & & & \\
\hline
\end{tabular}

Regarding borderline proteinuria, there was no difference between both groups ( $P=0.708)$, where $75 \%$ of the patients in $C G$ and $65 \%$ in TG had borderline proteinuria at a given moment (Fig. 1). As for true proteinuria, there was also no significant difference between the percentages of affected patients in either group $(P=0.588)$. In $C G, 50 \%$ of patients had proteinuria at some moment, while in $T G$, this number was $47 \%$ (Fig. 1).

There was no significant difference in development of chronic renal disease (CKD) between treatments $(P=0.894)$ or between moments $(P=0.929)$. The frequency for development of CKD was 33\% $(4 / 11)$ in CG and $39 \%(5 / 13)$ in TG (Fig. 2).

However, there was no difference in mortality between treatments $(P=0.422)$. Mortality rate was $39 \%$ $(5 / 13)$ and $46 \%(6 / 13)$ in control and treated group, respectively (Fig. 2), in which $23 \%(6 / 26)$ died at 
home, so a necropsy was not possible and the cause of death was undetermined; $8 \%(2 / 26)$ were euthanized due to metastasis in other organs; $4 \%(1 / 26)$ died with neurologic signs of undetermined origin; $4 \%(1 / 26)$ died in the immediate post-operative period; and $4 \%(1 / 26)$ died due to respiratory insufficiency from lung metastasis.

\section{Discussion}

The patients evaluated by B-mode ultrasound did not have significant morphologic changes related to structural kidney injury. The kidneys presented normal size and architecture, with homogeneous hypoechoic aspect compared to splenic parenchyma. Most patients had a preserved corticomedullary ratio. These results are in agreement with Bragato et al. (2015), who mentioned that diffuse renal changes can be hard to diagnose via B-mode ultrasound. When present, common changes would be nephromegaly, increased or decreased echogenicity of the renal cortex, renal pelvis dilation, among others.

Oyuela-Carrasco et al. (2009) observed that elderly patients may show an increase and later decrease in renal mass, which may be compatible with glomerulosclerosis and tubulointerstitial fibrosis, which in turn may lead to kidney injury. These results could explain the development of CKD observed in the present study, where the majority of the dogs were old, thus increasing the possibility that they already had some type of degenerative renal injury due to age, but without a correlation with kidney size.

In the present study, the parameters and indices evaluated by Doppler and CEUS did not show significant differences between groups or moments, and urea and creatinine levels remained within normal limits for the species throughout the experiment. Dong et al. (2013) described that CEUS can show changes in renal flow six weeks before creatinine values are significantly increased for the species, allowing an early diagnosis of renal diseases and their treatment. Similarly, Melo et al. (2006) and Martín et al. (2017) described the importance and reliability of Doppler flowmetry in identifying early changes in renal flow, anticipating irreversible structural damage to the kidneys. As such, the authors consider that there was no change in renal perfusion resulting from the treatment, and that the development of proteinuria and CKD were due to factors inherent to the patients and not the treatment.

Da Silva et al. (2014) reported that hematologic changes are common in patients with mammary neoplasia, often with presence of anaemia. They observed that $11 \%$ of bitches with mammary neoplasia had erythrogram correlated with tumour staging, where tumours in stage III, IV, and V had greater haematological changes. This study explains the present erythrogram findings, where patients had decreased erythrocyte count and haemoglobin at M0, but after surgery, these parameters gradually increased. This increase was significant starting at M3, which correlates with the absence of paraneoplastic anaemia in most patients after tumour removal.

Dietl et al. (2007) stated that between $50 \%$ and $70 \%$ of people with advanced stage neoplasia had anaemia caused by the tumour. However, De Oliveira et al. (2013) reported that changes in oncologic patient are not exclusively due to the presence of tumour or its location. Paraneoplastic syndromes may 
also be involved in hematologic changes, especially due to sequestering of iron and decrease in erythropoiesis and erythrocyte half-life. Surgical removal of the neoplasia is indicated as definitive treatment. The improvement seen on erythrograms in the present study supports these statements.

Figueiredo (2019) stated that inflammation is an important factor for neoplastic development and progression, and results from the tumour microenvironment composed of neoplastic cells, macrophages, neutrophils, lymphocytes, and other inflammatory cells, as well as cells that make up blood vessels, thus forming a pro-tumoral environment. The same author mentions that the presence of neutrophils and $B$ lymphocytes in the tumour microenvironment may aid tumour growth and be related to a worse prognosis. In the present study, the number of bands and lymphocytes were higher in most of the moments in control group, however, no relationship was observed with prognosis in these patients.

In this context, Forget et al. (2013) cited that an inflammatory state can be better evaluated based on the neutrophil/lymphocyte ratio (RNL) and this parameter was considered by various studies as an important prognostic factor in patients with different neoplasia, where mammary neoplasia was one of the most common. Jia et al. (2015) determined that pre-treatment RNL is a predictive indicator of disease-free survival and overall survival in patients with mammary neoplasia regardless of the tumour size or patients' age, where higher RNL values (>2.0) indicate a poor prognosis. Correlating these findings with those of the present study, RNL was lower in the treated group during second and third months, which may be related to the high mortality rate in control group in these moments. In spite of a high RNL in several moments in both groups, the absolute lymphocyte and band counts were lower in treated group. This response may be explained by the anti-inflammatory effect of carprofen, which would reduce local and systemic inflammatory process [19].

Cavalcante et al. (2008) mentioned that prolonged use of carprofen can affect liver enzymes, with an increase in ALT being more common, reporting 20 cases of liver toxicity due to carprofen administration, where 18 dogs showed signs of liver toxicity after receiving carprofen for 19 days and two dogs at 60 and 180 days. The main clinical signs observed in these patients were vomit, anorexia, and jaundice, and they all had an increase in ALT levels (above the normal range). In the present study, increased ALT was observed in patients treated with carprofen; however, ALT did not rise above the normal limits for the species. This was contrary to results obtained by Autefage \& Gossellin (2007), who administered carprofen to 110 dogs during 120 days and observed no clinical signs of liver toxicity or changes in liver enzyme levels during treatment.

Lucas et al. (2019) reported that NSAIDs can lead to acute or chronic kidney injury when used for prolonged periods. In the present study, no acute renal injury (azotaemia) was observed over six months when carprofen was given for 90 days. As for development of borderline and true proteinuria as markers of CKD, it was similar between groups, which highlights that the use of carprofen during the aforementioned period does not predispose to development of renal impairment. Results are in agreement with those obtained by Luna et al. (2007), who did not report renal changes in patients treated 
with carprofen during 90 days, by Raekallio et al. (2006) in patients treated during 60 days, and by Autefage \& Gosselin (2007) in patients treated during 120 days.

From a physiologic standpoint, when there is hypovolemia, the renin-angiotensin-aldosterone system is activated, causing vasoconstriction and an increase in sodium and water resorption. Prostaglandins (PGE2, PGD2) stimulate compensatory vasodilation of afferent arterioles and angiotensin II stimulates vasoconstriction of the efferent arterioles, thus improving renal blood flow and avoiding possible renal changes. Prostaglandins are products of the conversion of arachidonic acid from COXs, so in the context of kidney injuries due to administration of NSAIDS, it could be said that acute renal injury has a hemodynamic origin because of the limitation of this compensatory system $[25,26,27,28]$. This is the mechanism used by several studies $[26,27]$ to explain that the prolonged use of NSAIDS may result in the development of CKD; however, in the present study, this was not observed in association with administration of carprofen during 90 days within 180 days of follow-up.

It can be concluded that under the specified conditions, the use of carprofen for 90 days causes minimal changes in renal perfusion, red blood cell parameters, and ALT activity, reducing the amount of inflammatory cells in the blood, and does not result in kidney injury or change the mortality rate. As such, it is considered that carprofen can be used safely in patients that require auxiliary cancer treatment and in which its benefits will outweigh the side effects.

\section{Methods}

Ethical aspects. This prospective clinical study including all its methods followed the recommendations of the Brazilian National Council for the Control of Animal Experimentation (CONCEA) and were approved by the Ethics Committee in the Use of Animals of the São Paulo State University (Unesp), School of Agricultural and Veterinarian Sciences, Jaboticabal, São Paulo, Brazil (protocol no. 018989/17) and this study is in accordance with the arrive guideline. The tutors of the animals selected for this study were consulted, informed, and clarified regarding all details of the experiment and stated their agreement with the evaluations proposed in terms of free and informed consent. The selected patients were monitored during the experimental period and the veterinary team remained at the disposal for any intercurrence from the procedures.

Study design and animals and clinical assessment. This was a prospective clinical, case-control study conducted between March 2018 and November 2019. Twenty-six bitches (mean age $9.4 \pm 2.4$ years) with mammary carcinoma presented to the veterinary oncology service were selected. Inclusion criteria: animals previously diagnosed with grade III and IV breast carcinoma, with no evident changes on clinical and haematological examination that indicated liver or kidney disease. Exclusion criteria: having undergone any previous surgical procedure of the mammary gland, clinical signs of systemic diseases that compromised the treatment object of this study and/or anti-inflammatory treatment in the last six months. Physical evaluation, blood count, serum concentration of creatinine (Crea $\mathrm{mg} / \mathrm{dL})$, urea (mg/dL), alanine aminotransferase (ALT U/L), urinary analyses, urine protein/creatinine ratio (UPC), 
electrocardiogram were performed, thoracic radiographs and abdominal ultrasound were performed to investigate the presence of metastasis.

The patients were randomly distributed (via draw) into two experimental groups prior to receiving any medication or undergoing surgery (M0). The two groups were: treated group (TG) - treated with carprofen $(n=13)$; and control group (CG; $n=13)$.

The animals were submitted to radical unilateral mastectomy and ipsilateral axillar and inguinal lymphadenectomy. After discharge, cephalexin (30mg/kg/BID), omeprazole ( $1 \mathrm{mg} / \mathrm{kg} / \mathrm{BID})$, and tramadol hydrochloride $(3 \mathrm{mg} / \mathrm{kg} / \mathrm{TID})$ were prescribed orally in both groups. For histopathology examination, the biological material removed during mastectomy was fixed in an aqueous solution of $10 \%$ buffered formaldehyde and sent to the institution's veterinary pathology laboratory.

The dogs in the TG received orally carprofen $(4.4 \mathrm{mg} / \mathrm{kg})$ every 24 hours starting after surgery for 90 days. The CG did not receive any anti-inflammatory drugs during this period. For six months after surgery, all patients were re-examined every month (M1-M6) using the same diagnostic methods described for initial evaluation.

Ultrasound assessment. The patients were positioned in dorsal recumbency, a large area of their abdomen was shaved, and gel applied to the abdominal skin. The examination was performed by a single experienced operator who was blinded to the treatment group. The ultrasound device used was an Acuson S2000 (Siemens, Munich, Germany) with a 4.5 to $9.0 \mathrm{MHz}$ matrix linear transducer. During the exam, texture (homogeneous or heterogeneous), echogenicity (anechoic, hypoechoic, hyperechoic, or mixed), corticomedullary relation (CMR), kidney size (length, width, and height), and renal surface (regular or irregular) were evaluated on longitudinal and transverse views.

Mean area on transverse view of the left renal artery (RA) was obtained in triplicate during diastole and systole, and the kidneys measurements were used to calculate renal volume ( $R V=$ length $\mathrm{x}$ height $\mathrm{x}$ width $x$ 0.523) [7].

Colour Doppler was used to evaluate the integrity of renal vessels and to investigate the presence or absence of vascularized areas. Spectral Doppler was performed after locating the renal artery, and the Doppler angle along the long axis of the vessel was maintained at a maximum of $60^{\circ}$. Colour gain was adjusted to reduce excessive noise. The sampling window was regulated between 2 and $3 \mathrm{~mm}$ (equivalent to $2 / 3$ of the vessel diameter) and positioned over the central area of the vessel to automatically generate the spectral curve and calculate the vascular indices in three subsequent waves, systolic velocity (SV, cm/s), diastolic velocity (DV, cm/s), resistive index (RI), pulsatility index (IP), timeaveraged minimum velocity (TaMin) and time-averaged maximum velocity (TaMax). Doppler flowmetry parameters and RV and RA were used to calculate renal blood flow (RBF = (TaMax + TaMin / 2) $\times(R A /$ RV)), as described by Grunert et al. (1990) and Miyamoto et al. (1995). 
After Doppler examination, CEUS was performed using a harmonic imagining software (Cadence contrast pulse sequencing (CPS) technology - Siemens $\left.{ }^{\circledR}\right)$. The ultrasound image of the left kidney was centred on the screen, with the greatest possible renal length obtained on longitudinal view. Acoustic power (MI) was set at 0.09 , with gain, depth, dynamic range, frequency and focus optimized on initial evaluation to ensure excellent image quality and maintained throughout the experiment.

The perfluorocarbon ultrasound contrast SonoVue (Bracco $\left.{ }^{\circledR}\right)$ was administered as a bolus at $0.01 \mathrm{ml} / \mathrm{kg}$ intravenously, followed by a $5 \mathrm{ml}$ saline flush. The moment of injection was considered T0 and recording of the resulting images in video format was performed for 120 seconds for later analysis.

After acquisition, the images were transferred to an off-line analysis module (DICOM®), Digital Imaging and Communications in Medicine, National Electrical Manufacturers Association). Two trained evaluators, blinded to the treatment, analysed each of the image sequences obtained. The main areas of interest were defined in the renal cortex (Cort) and then medulla (Med). After automatic assembly of the image sequence, these were converted into time-intensity curves (TIC). Five subareas of interest (ROI) were drawn, each approximately $1 \mathrm{~mm}^{2}$. These ROI were located within the cortical or medullary parenchyma and were uniform in depth, as described by Wei et al. (2001). Based on the TIC, the processing software calculated the parameters for renal perfusion (cortical or medullary): peak intensity ( $\mathrm{PI}$ in \% of mean pixels), time to peak intensity (Tp in s), mean time of transmission (TmT in s), area under the curve (AUC), wash-in slope (a in Pixel/s), and wash-out slope (b in Pixel/s).

Statistical analysis. Statistical analyses were performed using R software (Foundation for Statistical Computing $\left.{ }^{\circledR}\right)$. Initially, normal distribution of the residues (Shapiro test) and homoscedasticity of the variances (variance test) were investigated for all studied parameters. The parametric variables were submitted to ANOVA with repeated measurements and Bonferroni post-hoc, where the following factors were compared: group, moments and its interaction. Non-parametric variables were analysed using the Friedman test and Dunn's post-hoc, for the same factors. Clinical and ultrasound parameters were correlated using Pearson or Spearman tests. Borderline and true proteinuria, chronic kidney disease (CKD) according to the criteria established by International Renal Interest Society (Elliott \& Cowgill 2017), and mortality in each studied group and moment were compared via Fisher's exact test and the Kaplan Meier survival method. Significance was fixed at $5 \%(p<0.05)$ for all tests.

\section{Declarations}

\section{Acknowledgements}

The authors would like to thank for National Council for Scientific and Technological (CNPq) for funding the research, Jair Matos from Siemens Healthinners for technical assistance, owners and breeders of the dogs participating in this study

\section{Author contributions}


C.R.V.E., R.A.R.U. and M.A.R.F. conceived, designed the study and participated in manuscript writing. C.R.V.E., B.F.F., D.B.V., M.C.M., R.A.R.U., B.G., M.A.R.F., L.P., L.P.N.A., G.P.L. and A.B.D.N executed the experiment, collected and interpreted data. R.A.R.U. Statistical analysed data. All authors interpreted the data, critically revised the manuscript for important intellectual contents and approved the final version.

\section{Competing interests}

The authors declare that they have no conflict of interest.

\section{References}

1. Pang, L. Y., Argyle, S. A., Kamida, A., Morrison, K. O. N. \& Argyle, D. J. The long-acting COX-2 inhibitor mavacoxib (Trocoxil ${ }^{\mathrm{TM}}$ ) has anti-proliferative and pro-apoptotic effects on canine cancer cell lines and cancer stem cells in vitro. BMC Veterinary Research. 10 (1), 184 (2014).

2. Doré, M., Lanthier, I. \& Sirois, J. Cyclooxygenase-2 expression in canine mammary tumors. Vet. Pathol. 40 (2), 207-212 (2003).

3. Doré, M. Cyclooxygenase-2 expression in animal cancers. Vet. Pathol. 48 (1), 254-265 (2011).

4. Berno, M. D. B. \& Mendes, R. Dor oncológica em pequenos animais - revisão de literatura. Revista Científica de Medicina Veterinária 2015. year XXIV, 1-16

5. Nogueira, A. C. et al. Ultra-sonografia com agentes de contrastes por microbolhas na avaliação da perfusão renal em indivíduos normais. Revista Brasileira de Ecocardiografia. v.15, 74-78 (2002).

6. Melo, M. B., Veado, J. C. C., Silva, E. F., Moreira, S. M. \& Passos, L. M. F. Dopplerfluxometria das artérias renais: valores normais das velocidades sistólica e diastólica e do índice resistivo nas artérias renais principais. Brazilian Journal of Veterinary and Animal Science. 58 (4), 691-693 (2006).

7. Grunert, D., Schöning, M. \& Rosendahl, W. Renal blood flow and flow velocity in children and adolescents: Duplex Doppler evaluation. European Journal of Pediatrics. 149 (4), 287-292 (1990).

8. Miyamoto, T. et al. Quantitative measurement of canine renal arterial blood flow using Doppler ultrasonography. Journal of Veterinary Medical Science. 57 (4), 785-788 (1995).

9. Bragato, N., Fioravanti, M. C. S., Braga, L. G., Reis, D. C. D. \& Borges, N. C. Lesão renal tubular aguda em cães e gatos: fisiopatogenia e diagnóstico ultrassonográfico. Enciclopédia Biosfera. 11, 2092 (2015).

10. Oyuela-Carrasco, J., Rodríguez-Castellanos, F., Kimura, E., Delgado, R. E. \& Herrera-Felix, J. P. Longitud renal por ultrasonografía en población mexicana adulta. Nefrología. 29 (1), 30-34 (2009).

11. Dong, Y., Wang, W., Cao, J., Fan, P. \& Lin, X. Quantitative evaluation of contrast-enhanced ultrasonography in the diagnosis of chronic ischemic renal disease in a dog model. Plos One. 8 (8), (2013). e70337

12. Martín, C. M., Kogika, M. M., Miyashiro, S. I. \& Fonseca-Pinto, A. C. B. D. C. Ultrassonografia modo B e Doppler na avaliação renal de cães submetidos à tomografia computadorizada após administração 
intravenosa de diferentes meios de contraste iodado. Pesquisa Veterinária Brasileira. 37 (7), 759772 (2017).

13. Da Silva, A. H. C. et al. Alterações no hemograma de cadelas com neoplasia mamária. Ciência Animal Brasileira. 15 (1), 87-92 (2014).

14. Dietl, B., Marienhagen, J., Schäfer, C. \& Kölbl, O. The prognostic value of anaemia at different treatment times in patients with locally advanced head and neck cancer treated with surgery and postoperative radiotherapy. Clin. Oncol. 19 (4), 228-233 (2007).

15. De Oliveira, K. M., Dos Santos Horta, R., Silva, C. M. O. \& Lavor M. S. L. Principais síndromes paraneoplásicas em cães e gatos. Enciclopédia biosfera 2013. 9,2073

16. Figueiredo, C. R. L. The unusual paradox of cancer-associated inflammation: an update. Jornal Brasileiro de Patologia e Medicina Laboratorial. 55 (3), 321-332 (2019).

17. Forget, P. et al. Neutrophil: lymphocyte ratio and intraoperative use of ketorolac or diclofenac are prognostic factors in different cohorts of patients undergoing breast, lung, and kidney cancer surgery. Annals of surgical oncology 2013. 20 (3),650-660. DOI: 10.1245 / s10434-013-3136-x

18. Jia, W. et al. The peripheral blood neutrophil-to-lymphocyte ratio is superior to the lymphocyte-tomonocyte ratio for predicting the long-term survival of triple-negative breast cancer patients. Plos One 2015. 10 (11), e.0143061.

19. Pimpão, C. T. et al. Avaliação do carprofeno e do meloxicam como antipiréticos em cães. Revista Acadêmica Ciência Animal. 7 (3), 331-339 (2009).

20. Cavalcante, L. F. H., Marques, J. M. V., Gouvêa, A. S. \& Contesini, E. A. Intoxicação por carprofeno em cães - artigo de revisão. Revista da FZVA. 15 (1), 160-171 (2008).

21. Autefage, A. \& Gosselin, J. Efficacy and safety of the long-term oral administration of carprofen in the treatment of osteoarthritis in dogs. Revue de Médecine Vétérinaire. 1 (3), 119-127 (2007).

22. Lucas, G. N. C. et al. Pathophysiological aspects of nephropathy caused by non-steroidal antiinflammatory drugs. Brazilian Journal of Nephrology. 41 (1), 124-130 (2019).

23. Luna, S. P. L. et al. Evaluation of adverse effects of long-term oral administration of carprofen, etodolac, flunixin meglumine, ketoprofen, and meloxicam in dogs. American Journal of Veterinary Research. 68 (3), 258-264 (2007).

24. Raekallio, M. R., Hielm-Björkman, A. K., Kejonen, J., Salonen, H. M. \& Sankari, S. M. Evaluation of adverse effects of long-term orally administered carprofen in dogs. Journal of the American Veterinary Medical Association. 228 (6), 876-880 (2006).

25. Ulinski, T., Guigonis, V., Dunan, O. \& Bensman, A. Acute renal failure after treatment with non-steroidal anti-inflammatory drugs. European Journal of Pediatrics. 163 (3), 148-150 (2004).

26. Monteiro, E. C. A., Trindade, J. M. D. F., Duarte, A. L. B. P. \& Chahade, W. H. Os antiinflamatórios não esteroidais (AINEs). Temas de reumatologia clínica 2008. 9 (2),53-63

27. Melgaço, S. S. C., Saraiva, M. I. R., Lima, T. T. C., Júnior, G. B. S. \& Daher, E. F. Nefrotoxicidade dos anti-inflamatórios não esteroidais. Medicina (Ribeirao Preto. Online). 43 (4), 382-390 (2010). 
28. Michellin, A. F., Ferreira, A. A. P., Bitar, V. G. \& Lopes, L. C. Toxicidade renal de inibidores seletivos da ciclooxigenase-2: celecoxib e rofecoxib. Revista de Ciências Médicas 2012. 15 (4),321-332

\section{Figures}
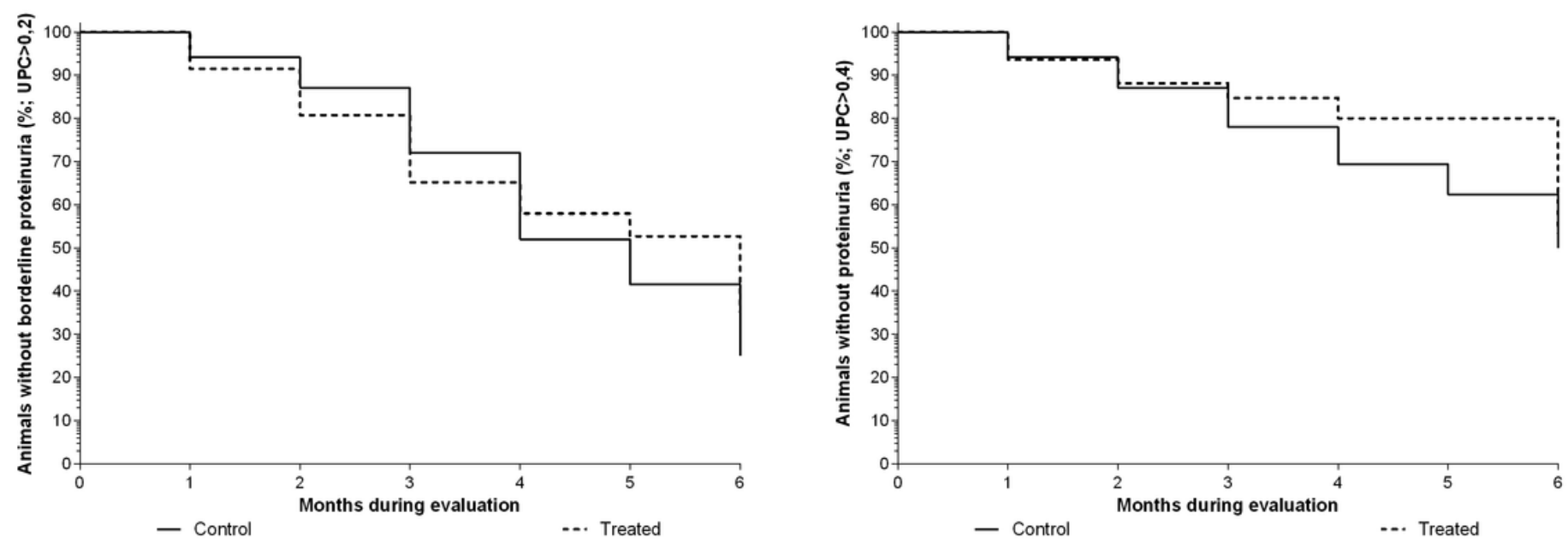

\section{Figure 1}

Kaplan Maier survival curves for analysis of borderline proteinuria (left) and true proteinuria (right) in bitches with mammary carcinoma treated (TG) or not treated (CG) with carprofen at $4.4 \mathrm{mg} / \mathrm{kg} / \mathrm{day}$ for 90 days, during a 6-month follow-up.
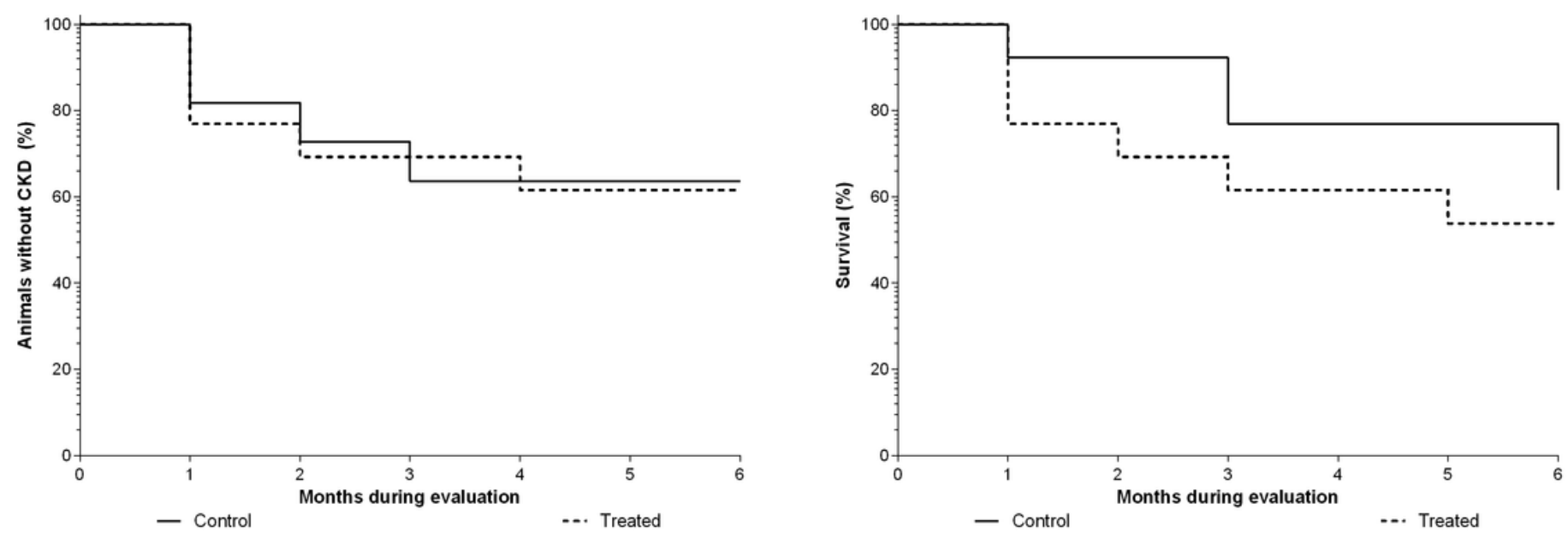

Figure 2

Kaplan Maier survival curves for analysis of chronic renal insufficiency (CKD, left) and mortality (right), in bitches with mammary carcinoma with (TG) or without (CG) carprofen at $4.4 \mathrm{mg} / \mathrm{kg} / \mathrm{day}$ for 90 days, evaluated during 6 months. 